\section{A case of chronic progressive Lyme encephalitis as a manifestation of late Lyme neuroborreliosis}

\author{
Vivek Verma, ${ }^{1}$ Matthew Roman, ${ }^{1}$ \\ Disha Shah, ${ }^{2}$ Marina Zaretskaya, ${ }^{2}$ \\ Mohamed H. Yassin ${ }^{1,3}$ \\ 'Department of Medicine, ${ }^{2}$ Division \\ of Neurology, ${ }^{3}$ Division of Infectious \\ Diseases, University of Pittsburgh \\ Medical Center, PA, USA
}

\begin{abstract}
A 54-year-old female living in Europe presented with gait ataxia, dizziness, and bilateral hearing loss. Magnetic resonance imaging (MRI) revealed non-specific white matter changes. The patient's condition gradually deteriorated over two years without diagnosis. The patient continued to decline cognitively and neurologically with worsening ataxia and upper motor neuron signs. Repeat MRI showed worsening white matter changes. Lumbar puncture, not previously done, showed positive Lyme testing. Treatment with intravenous ceftriaxone resulted in marked neurological improvement. Four years after symptom, the patient has short-term memory deficits and chronic fatigue, but is otherwise neurologically, cognitively, and functionally intact. Follow up MRI findings remain largely unchanged. Because cases of intraparenchymal or encephalopathic neuroborreliosis in America are lacking, so are treatment options. We present a rare case and discuss our experience with antibiotic treatment. This case lends evidence to define optimal treatment of this disease, imperative for hastening neurological recovery.
\end{abstract}

\section{Introduction}

Lyme neuroborreliosis (LNB) is an infectious disorder of the nervous system caused by tick-borne spirochetes of the Borrelia burgdorferi sensu lato complex. It can commonly start with a bull's eye rash (erythema migrans) and can systemically spread, causing arthritis, heart block, and effects in both the central and peripheral nervous systems. ${ }^{1}$ In Europe, LNB is most often caused by $B$. garinii, less frequently by $B$. afzelii, and rarely by $B$. burgdorferi sensu stricto (which is predominantly in North America). ${ }^{2}$ Borrelia subspecies are thus responsible for significant differences in the clinical presentations of LNB observed in
North America and Europe. Early LNB can cause lymphocytic meningitis; involvement of cranial and peripheral nerves is reported to occur in $5-15 \%$ of untreated patients. ${ }^{2}$ Late LNB, which is considerably rarer, can involve brain/spinal cord parenchymal inflammation. ${ }^{2,3}$

There are important clinical consequences of these different species and their geographic distributions, possibly as a result of differential species pathogenicity, as illustrated in one study. ${ }^{4}$ Neuroborreliosis is the most common disseminated infection of Lyme disease in Europe, but is infrequently reported in the United States. Furthermore, even though all three aforementioned subspecies exist in Europe, the same study notes that the North American-predominant subspecies (B. burgdorferi sensu stricto) is responsible for only $8-11 \%$ of neuroborreliosis cases in Europe. ${ }^{4}$ Thus, this could explain the fact that reports of neuroborreliosis reported in the US (particularly with encephalopathy, for instance) are rare, especially in the adult population. As a result, optimal treatment regimens of central nervous system (CNS) parenchymal neuroborreliosis are incomplete, according to the American Academy of Neurology (AAN). ${ }^{3}$ The present case describes a patient with chronic Lyme neuroborreliosis; potentially adding much-needed evidence on effective management of this rare but serious disease.

\section{Case Report}

A 54-year-old Caucasian female with past medical history of uncomplicated migraines first noticed dizziness and ataxic gait in April 2009. The woman, American by nationality, was living with her husband in the Netherlands at the time. Shortly thereafter, she developed bilateral hearing loss and noticed a 20-pound unintentional weight loss over the previous couple months. She was admitted to a hospital in the Netherlands, where reportedly blood work, electroencephalography (EEG), and echocardiogram were normal. Magnetic resonance imaging (MRI) at the time apparently showed nonspecific white matter changes present over both cerebral hemispheres. The patient remained relatively stable until her next episode that occurred in April 2011. A new, sudden-onset gait imbalance triggered further workup. Though magnetic resonance angiography (MRA) was normal, MRI was initially read as being suspicious of an apparent small, acute non-hemorrhagic stroke in the left cerebellar peduncle as well as progressive white matter changes (on a reviewed re-read, it was not read as a stroke). EEG showed sharp waves over the left temporal lobe but no foci of epilep-
Correspondence: Mohamed H. Yassin, University of Pittsburgh Medical Center, Mercy Hospital, 10550 Ermire Building, 1400 Locust Street, Pittsburgh, PA 15219, USA.

Tel.: +1.412.232.7798 - Fax: +1.412.232.3292

E-mail: yassinm@upmc.edu

Key words: Lyme neuroborreliosis, Borrelia burgdorferi, encephalopathy.

Contributions: VV and MR analyzed data and patient records, obtained references, prepared figures and table, and overall wrote the manuscript; MZ and MHY supervised the aforementioned and took care of the patient in this case report in an inpatient and outpatient setting.

Conflict of interests: the authors declare no potential conflict of interest.

Received for publication: 21 July 2014.

Revision received: 30 September 2014.

Accepted for publication: 30 September 2014.

This work is licensed under a Creative Commons Attribution NonCommercial 3.0 License (CC BYNC 3.0).

(C) Copyright V. Verma et al., 2014

Licensee PAGEPress, Italy

Infectious Disease Reports 2014; 6:5496

doi:10.4081/idr.2014.5696

tiform activity. Her workup was otherwise unrevealing; it included complete blood count, basic metabolic panel, creatine phosphokinase, lactate, echocardiogram with bubble study, chest radiograph, vasculitis and hypercoagulable profiles. At that time, she was started on aspirin $81 \mathrm{mg}$ daily as well as levetiracetam $250 \mathrm{mg}$ twice daily.

The patient was then referred to our facility for neurological evaluation. The patient reported subjective generalized weakness/fatigue, but denied any fevers, rash, joint pains, swallowing difficulty, or visual problems. She had no family history of any neurological disorders. Her examination revealed slowed speech, bilateral decrease in hearing, and bilateral non-sustained nystagmus on horizontal gaze. She was alert and oriented times three, but had poor cognition and insight into her disease history. Bilateral dysmetria, ataxic gait, hyperreflexia with nonsustained clonus and bilateral Babinski reflexes were also found on exam. Repeat brain MRI at our facility (Figure 1) showed relatively unchanged scattered round-oval hyperintensities in the subcortical and periventricular white matter, as well as cerebellum and pons. Spinal MRI did not show any lesions. $\mathrm{B}_{12}$ level was low-normal at 242 $\mathrm{pg} / \mathrm{mL}$ (normal range 200-900), for which she was empirically started on weekly subcutaneous $B_{12}$ injections ( $\left.1000 \mathrm{mcg}\right)$ to make sure $\mathrm{B}_{12}$ levels did not drop and potentially com- 
pound symptoms. Lumbar puncture (LP) done at that time revealed normal opening pressure with 200 white blood cells (65\% monocytes, $35 \%$ neutrophils), zero red blood cells, low glucose level of 11 (serum level 107), and elevated protein $324 \mathrm{mg} / \mathrm{dl}$ (normal range 15-45).

The patient was admitted to the hospital and the infectious diseases service was consulted. Repeat LP two days post-admission showed similar results (yellow-colored fluid, 229 white blood cells (55\% monocytes, $45 \%$ neutrophils), 12 red blood cells, glucose 14, protein 290). Autoimmune workup was negative for antinuclear antibody, antineutrophil cytoplasmic antibody, rheumatoid factor; angiotensin-converting enzyme, aldolase, erythrocyte sedimentation rate, and C-reactive protein levels were normal. Antibodies to cardiolipin, myeloperoxidase-3, proteinase-3, Sjogren-A/B, and paraneoplastic antibodies against neuronal nuclei and Purkinje cells anti-neuronal nuclear antibody (ANNA), and Purkinje cell cytoplasmic antibody (PCCA) were negative. Infectious diseases workup that was negative is listed in Table 1. Screening cerebrospinal fluid (CSF) enzyme-linked immunosorbent assay (ELISA) for Lyme disease was positive at 4.42 (reference range $<1.1$ ). A confirmatory Western blot subsequently revealed positive bands at 18,23 , 41 , and $58 \mathrm{kDa}$. Though antibodies were detected in the CSF, polymerase chain reaction (PCR) for Lyme DNA in CSF was negative times two. The overall CSF immunoglobulin (IgG) level was elevated to 65.6 (reference range 0.8 to 7.7$)$, as was the IgG index ( 0.89 , normal <0.6) with oligoclonal bands. CSF myelin basic protein was normal, however. All CSF cultures for bacteria (including mycobacteria) and fungi remained negative.

On review of these results, the patient was diagnosed with LNB. IV ceftriaxone $2 \mathrm{~g}$ daily was administered via a peripherally inserted intravenous catheter (PICC) for 28 days. The patient finished her course of ceftriaxone and followed up as outpatient with both Neurology and Infectious Diseases. After her antibiotic course, the patient noted complete resolution of her ataxia, dizziness, hearing loss, and improvement of weakness/fatigue. Levetiracetam was stopped in March 2012. At the time of last follow-up (March 2013), almost four years after initial symptoms, the patient's only reported symptoms were chronic fatigue (much improved relative to before antibiotics started), and some impairment in formation of short-term memories. On exam, she does display $0 / 3$ word recall at five minutes, slightly decreased fine motor coordination, and is slightly hyperreflexic (albeit markedly less so compared to initial exam). Otherwise she remains neurologically, cognitively, and functionally intact. Follow up brain MRI showed slight decrease in size of the pontine and cerebellar peduncular lesions, but otherwise no changes (Figure 2).
Table 1. Negative infectious disease workup in this patient.

\begin{tabular}{ll}
1 & Herpes simplex virus-1 and -2 PCR, CSF \\
2 & Epstein-Barr virus PCR, CSF \\
\hline 3 & Cytomegalovirus PCR, CSF \\
\hline 5 & Mycobacterial stain and culture, CSF \\
\hline 6 & Syphilis, CSF \\
\hline 7 & Cryptococcus antigen, CSF \\
\hline 8 & Histoplasma antigen, urine \\
\hline 10 & HIV ELISA, serum \\
\hline 11 & West Nile virus serology, CSF \\
12 & Lymphocytic choriomeningitis virus serology, CSF \\
\hline 13 & Coxsackievirus serology panel, CSF \\
14 & Mumps serology, CSF \\
\hline 15 & St. Louis encephalitis serology, CSF \\
\hline 16 & Eastern/Western equine encephalitis serology, CSF \\
\hline 17 & California encephalitis serology, CSF \\
\hline 18 & Echovirus serology, CSF \\
\hline 19 & Adenovirus serology, CSF \\
\hline PCR, polymerase chain reaction; CSF, cerebrospinal fluid.
\end{tabular}
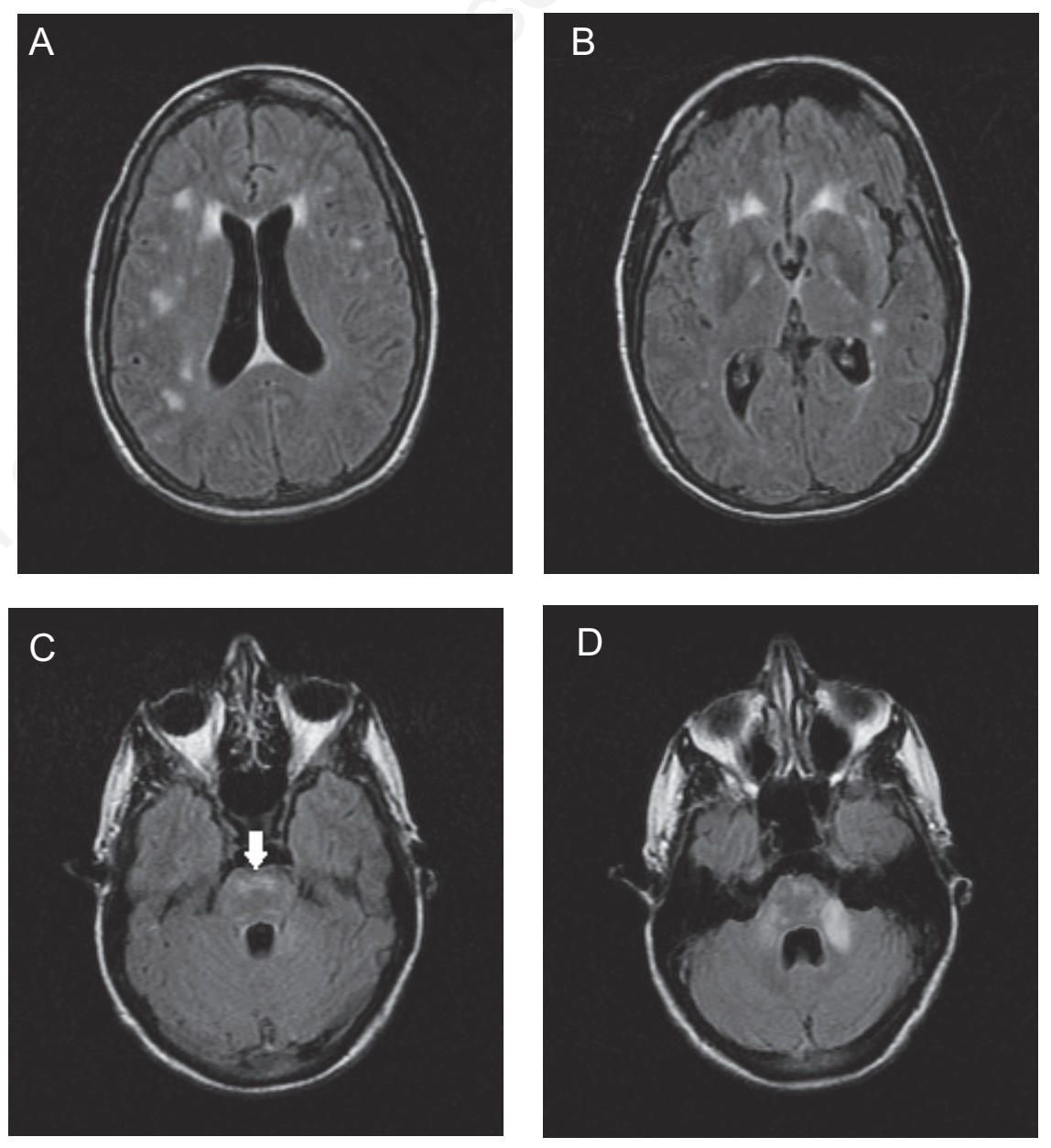

Figure 1. Fluid-attenuated inversion recovery - fast spin echo sequence images of the patient's brain on initial presentation to our hospital. Lesions are present in the periventricular areas $(\mathrm{A})$, white matter $(\mathrm{A}, \mathrm{B})$, pons $(\mathrm{C}$, arrow), and middle cerebellar peduncle (left $>$ right, D). 


\section{Discussion}

The present patient was probably infected with a European-specific species of Borrelia (B. garinii or B. afzelii); although European in origin, this case adds data to the overall data that is reported in the United States. Though the causative agent was not specifically genotyped, it would not have changed clinical management diagnostically nor therapeutically.

On radiological review, the main item on the differential diagnosis of the patient's MRI lesions, and certainly more common in North America, included demyelinating conditions. Hence, multiple sclerosis (MS) should be at the forefront of a differential diagnosis of similar brain lesions, as should other infectious causes (e.g., syphilis and other infectious causes in Table 1). Vitamin $B_{12}$ deficiency and autoimmune conditions such as systemic lupus erythematosus are important to consider, as well as the rare but frequently missed acute disseminated encephalomyelitis. ${ }^{5}$
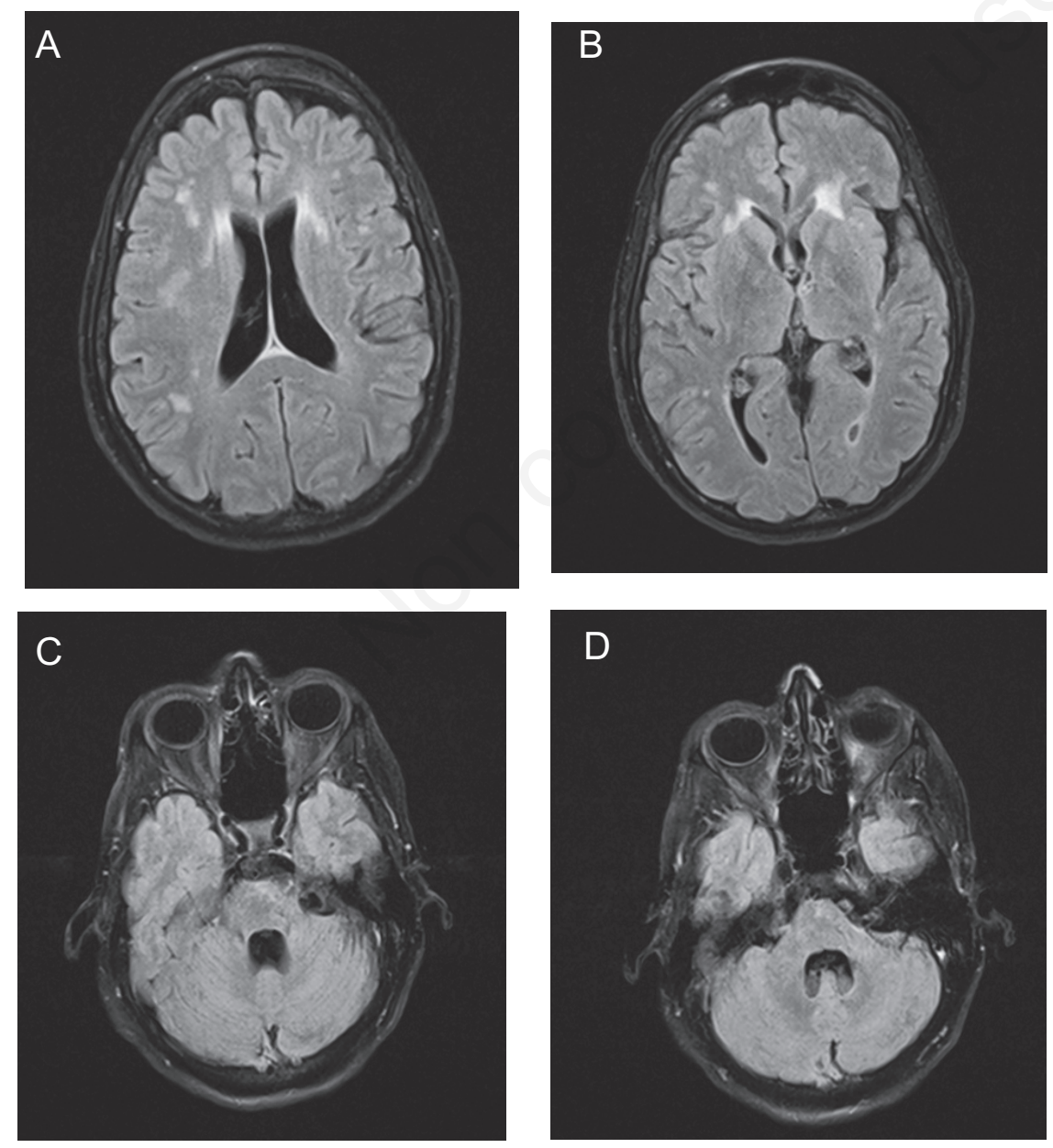

Figure 2. Fluid-attenuated inversion recovery - conventional spin echo sequence images of the patient's brain at two-year follow-up. Lesions persist in the periventricular areas and white matter $(A, B)$, but show decreased signal in the pons $(C)$ and middle cerebellar peduncle (D) relative to corresponding images in Figure 1. differ from MS lesions that are largely around CSF/ventricular flow. Most importantly, the patient's clinical status vastly and rapidly improved within weeks of IV antibiotic therapy without any relapse or new symptoms at two year follow up. ${ }^{9}$ Chronically progressing neurological symptoms that disappeared without exacerbation after antibiotic use (no corticosteroids or newer anti-inflammatory MS agents were used) further points to LNB over MS. Overall, a salient point to glean from this clinical diagnostic process is that often in clinical medicine, some items on the differential diagnosis can never be fully excluded (e.g. recurrent or multiphasic disseminated encephalomyelitis), but based on a constellation of many diverse supporting factors (in this patient, multiple laboratory tests, clinical picture, radiology, and treatment course), a principal diagnosis can indeed be reached.

Diagnosis in this patient was highly suspected based the presence of intrathecal antiBorrelia antibodies. In accordance with both the European Federation of Neurological Societies and the AAN, the gold standard of LNB diagnosis involves intrathecal antiBorrelia antibodies together with the clinical patient picture. ${ }^{9,12}$ Though serum Lyme serology was not checked due to initial suspicion for MS, both aforementioned guidelines endorse that clinically diagnosing LNB is essential. The patient's rapid and relapse-free improvement for two years after IV ceftriaxone further supports the diagnosis. Additionally, it was uncertain why hypoglycorrhachia was present, which is atypical of LNB, but it could possibly be related to the relatively long duration of the illness in this patient before the diagnosis was made.

At the time of follow-up, neither Lyme serology nor repeat lumbar puncture for monitoring intrathecal antibodies was done. These have been shown to be of relatively lower yield as follow-up measures because they can be persistently elevated for years after treatment, irrespective of symptomatic remission. ${ }^{13,14}$ Recent research, however, has helped to identify biomarkers such as CXCL13 with high sensitivity and specificity to identify acute LNB as well as monitor during follow-up. ${ }^{15}$ This is especially important in rare cases without intrathecal antibody production to cement a diagnosis without perpetuating neurological symptoms. Though further work will need to be done, novel biomarkers remain intriguing options as diagnostic aids for this disease.

It is also noteworthy that at most recent (2 year) follow-up, the patient's MRI signals have not changed for the most part (only the signal abnormalities in the pons and cerebellar peduncle improved). More importantly, as long as the patient's clinical status is (and remains) improved and nearly intact, this is in our opinion of little consequence. It could be 
possible that because this patient had chronic disease, and/or the nature of the infection, the lesions may never change despite the patient being clinically normal. Indeed, there are radiology reports that support this notion, including one with a five-year follow-up. ${ }^{16}$

This case supports the use of parenteral antibiotics to treat this patient, since the patient had an excellent clinical response with $2 \mathrm{~g}$ IV ceftriaxone. In terms of antibiotic therapy, our treatment is consistent with recommendations from the Infectious Diseases Society of America and the AAN, though the latter report mentions that there is a dearth of supportive data for cases involving the CNS parenchyma. $^{3,17}$ Though we continued Levetiracetam for seizure prophylaxis in this patient, we did not use corticosteroids; it is apparent that more research needs to be done on this issue, although the AAN hints that there may be no major impact of using corticosteroids. ${ }^{3}$ To prevent future cases of delayed diagnosis while the patient has continuing neurological symptoms, it is important for clinicians to keep Lyme disease on the differential diagnosis of any lingering neurological symptoms. This pathogen, possibly depending on the strain, can be very difficult to clear by the immune system. Indeed, animal studies have showed that if Borrelia can survive the initial innate immune response, there are very few barriers to developing long-term infection within distant tissues such as the brain. ${ }^{18}$ Clinically, this means Lyme disease should be high on a lingering neurological disease differential diagnosis.

Lastly, in patients with documented history of LNB, there are salient complications for clinicians to regularly monitor, which this patient displayed as well. As many as $15 \%$ of patients can experience chronic symptoms (such as fatigue, depression, memory or concentration defects, and musculoskeletal pain) which can occur years after completion of therapy, despite positive clinical response to initial antibiotic therapy. ${ }^{10,19}$ Unfortunately, these symptoms do not respond to additional antibiotics, so their administration is largely not warranted; management is best done symptomatically and with gentle reassurance/counseling. ${ }^{10}$ It is also important for physicians to not completely ignore recurrence of Lyme-specific symptoms (such as erythema migrans), because a small proportion of patients develop recurrent Lyme disease, which is thought to be due to reinfection with a different strain rather than relapse from the previous strain..$^{20}$ These cases would be managed with repeat antibiotics; management would be as if the patient were Lyme-naïve.

\section{Conclusions}

The present case serves to show that a patient presenting with many neurological deficits not localized to one area must be investigated thoroughly to rule out rare diseases such as LNB. In suspected cases, though data is greatly lacking in the USA, doing a prompt and deep investigation (also ruling out other causes on the differential diagnosis such as multiple sclerosis) is important to provide early treatment for the patient instead of causing lingering and possibly additive neurological deficits. Once diagnosed, however, our experience shows that parenteral antibiotic treatment works quite effectively for near-complete resolution of clinical symptoms, regardless of the predictable persistence of radiologic findings.

\section{References}

1. Stanek G, Wormser GP, Gray J, Strle F. Lyme borreliosis. Lancet 2012;379:461-73.

2. Strle F, Stanek G. Clinical manifestations and diagnosis of Lyme borreliosis. Curr Probl Dermatol 2009;37:51-110.

3. Halperin JJ, Shapiro ED, Logigian E, et al. Practice parameter: treatment of nervous system Lyme disease (an evidence-based review): report of the Quality Standards Subcommittee of the American Academy of Neurology. Neurology 2007;69:91-102.

4. Wang G, van Dam AP, Schwartz I, Dankert J. Molecular typing of Borrelia burgdorferi sensu lato: taxonomic, epidemiological, and clinical implications. Clin Microbiol Rev 1999;12:633-53.

5. Rocha R, Lisboa L, Neves J, et al. Neuroborreliosis presenting as acute disseminated encephalomyelitis. Pediatr Emer Care 2012;28:1374-6.

6. Hansen K, Cruz M, Link H. Oligoclonal Borrelia burgdorferi-specific IgG antibodies in cerebrospinal fluid in Lyme neuroborreliosis. J Infect Dis 1990;161:1194202.

7. Schmutzhard E. Multiple sclerosis and Lyme borreliosis. Wien Klin Wochenschr 2002;114:539-43.

8. Heller J, Holzer G, Schimrigk K.
Immunological differentiation between neuroborreliosis and multiple sclerosis. $\mathrm{J}$ Neurol 1990;237:465-70.

9. Mygland $\AA$, Ljøstad U, Fingerle V, et al. EFNS guidelines on the diagnosis and management of European Lyme neuroborreliosis. Eur J Neurol 2010;17:8-16.

10. Feder HM Jr, Johnson BJB, O'Connell S, et al. A critical appraisal of chronic Lyme disease. N Engl J Med 2007;357:1422-30.

11. Miller DH, Kesselring J, McDonald I, et al. Magnetic resonance in multiple sclerosis. $1^{\text {st }}$ ed. Cambridge, UK: Cambridge University Press; 1997.

12. Halperin JJ, Logigian EL, Finkel MF, Pearl RA. Practice parameters for the diagnosis of patients with nervous system Lyme borreliosis (Lyme disease). Neurology 1996;46:619-27.

13. Hammers-Berggren S, Hansen $\mathrm{K}$, Lebech AM, Karlsson M. Borrelia burgdorferi-specific intrathecal antibody production in neuroborreliosis. Neurology 1993;43:16975 .

14. Hammers-Berggren S, Lebech AM, Karlsson M, et al. Serological follow-up after treatment of patients with erythema migrans and neuroborreliosis. J Clin Microbiol 1994;32:1519-25.

15. Borde JP, Meier S, Fingerle V, et al. CXCL13 may improve diagnosis in early neuroborreliosis with atypical laboratory findings. BMC Infect Dis 2012;12:344.

16. Hildenbrand $\mathrm{P}$, Craven DE, Jones R, Nemeskal P. Lyme neuroborreliosis: manifestations of a rapidly emerging zoonosis. Am J Neuroradiol 2009;30:1079-87.

17. Wormser GP, Dattwyler RJ, Shapiro ED, et al. The clinical assessment, treatment, and prevention of Lyme disease, human granulocytic anaplasmosis, and babesiosis: clinical practice guidelines by the Infectious Diseases Society of America. Clin Infect Dis 2006;43:1089-134.

18. Troy EB, Lin T, Gao L, et al. Understanding barriers to Borrelia burgdorferi dissemination during infection using massively parallel sequencing. Infect Immun 2013;81:2347-57.

19. Eikeland R, Mygland $\AA$, Herlofson K, Ljøstad U. European neuroborreliosis: quality of life 30 months after treatment. Acta Neurol Scand 2011;124:349-54.

20. Nadelman RB, Hanincova K, Mukherjee P, et al. Differentiation of reinfection from relapse in recurrent Lyme disease. $\mathrm{N}$ Engl J Med 2012;367:1883-90. 